\title{
Técnicas de Aprendizagem Curricular aplicadas ao Diagnóstico Precoce do Câncer de Pele
}

\author{
Luís F. H. Serrano*, Sandra Avila
}

\begin{abstract}
Resumo
Detectar precocemente o câncer de pele é crucial: a taxa de sobrevivência é muito alta — cerca de $95 \%$ - para o diagnóstico precoce, mas cai substancialmente - para 10\% a 15\% - se o câncer atingir seus estágios finais. Neste contexto, o principal objetivo deste trabalho é facilitar a identificação precoce das lesões, melhorando dessa forma o prognóstico da doença. Para isso, focamos nas arquiteturas de aprendizado profundo (DLA, Deep Learning Architectures). Entretanto, o uso das DLAs para imagens médicas é problemático, devido à escassez de grandes bases de dados disponibilizadas. A utilização de tais dados pode ser melhorada a partir da aprendizagem curricular, uma estratégia de ordenação inteligente de tais dados que facilita o processo de aprendizagem.
\end{abstract}

\section{Palavras-chave: \\ Aprendizagem profunda, Aprendizagem curricular, Imagens médicas}

\section{Introdução}

Arquiteturas de aprendizado profundo (DLAs) são o estado da arte para tarefas de classificação que podem contar com grandes conjuntos de treino anotados. O diagnóstico assistido por computador, no entanto, apresenta sérios desafios devido à escassez de dados anotados, e a sutileza da diferença entre as imagens positivas/malignas e as negativas/benignas.

Esse trabalho visou investigar e aprimorar a classificação automática de imagens de câncer de pele, por meio da aplicação da aprendizagem curricular (curriculum learning) [1], uma estratégia de ordenação inteligente dos dados que facilita o processo de aprendizagem.

\section{Resultados e Discussão}

A ordem em que dados de treinamento são fornecidos às DLAs pode ser denominado de currículo. Tipicamente, este currículo é ordenado de forma aleatória e envolve todo o conjunto de treino. Contudo, humanos e animais aprendem mais facilmente e melhor quando são ensinados por exemplos de complexidade crescente. A estratégia de aprendizagem curricular emula este processo através da geração de um currículo adequado à DLA em questão. Esta estratégia originou diversas abordagens de criação de currículos.

Neste trabalho investigamos duas abordagens de aprendizagem curricular: ScreenerNet [2] e Conjuntos de Dados Crescentes Aleatórios [3].

O ScreenerNet utiliza uma segunda rede neural, que é treinada junto da rede original, para determinar quão relevante é cada exemplo para o treinamento naquele momento.

Os Conjuntos de Dados Crescentes Aleatórios (ROGS, Random Ordered Growing Sets) são utilizados para realizar um treinamento dividido em estágios, inicialmente sendo somente uma fração do conjunto original, mas expandido até se tornar o conjunto inteiro.

Primeiramente, avaliamos o ScreenerNet. Os resultados (não reportados aqui) não foram satisfatórios, uma vez que utilizando o código fornecido pelos autores não foi possível reproduzir seus resultados.

Em seguida foram feitos experimentos utilizando a técnica de ROGS. Os resultados foram mais consistentes ao se garantir que a proporção de classes diferentes era a mesma em todos os estágios (cada estágio contém parte do conjunto de treinamento). O treinamento de um estágio é concluído quando o erro medido num conjunto de validação não melhora por um certo número de épocas sucessivas (tal número é nomeado paciência). Os resultados estão na Tabela 1. A rede utilizada no treinamento é uma adaptação da utilizada por Menegola et al. [4], a rede Inception-v4.

Tabela 1. Resultados (em AUC) para a abordagem Conjuntos de Dados Crescentes Aleatórios no conjunto ISIC Challenge 2017.

\begin{tabular}{|l|l|l|l|l|l|l|}
\hline Est. & Pac. & Teste1 & Teste2 & Teste3 & Teste4 & Teste5 \\
\hline 1 & 20 & 0.858 & 0.899 & 0.871 & 0.886 & 0.892 \\
\hline 3 & 3 & 0.864 & 0.836 & 0.849 & 0.856 & 0.849 \\
\hline 3 & 6 & 0.893 & 0.886 & 0.850 & 0.891 & 0.845 \\
\hline 5 & 3 & 0.849 & 0.880 & 0.889 & 0.867 & 0.883 \\
\hline 5 & 6 & 0.880 & 0.854 & 0.872 & 0.869 & 0.866 \\
\hline
\end{tabular}

\section{Conclusão}

Apesar dos resultados não satisfatórios, é possível que uma configuração de ROGS com mais estágios ou distribuição de classes diferente traga melhores resultados, ou a utilização de outra técnica, como a aprendizagem autodirigida invertida.

\section{Agradecimentos}

Agradecemos ao PIBIC/CNPq (\#170181/2017-0) pelo financiamento da pesquisa.

\footnotetext{
${ }^{1}$ Bengio, Y., et al., "Curriculum learning," in International Conference on Machine Learning, 2009, pp. 41-48.

2 Kim, T., and Choi, J., "ScreenerNet: Learning curriculum for neural networks,” arXiv, abs/1801.00904, 2018.

${ }^{3}$ Mermer, M., and Amasyali, M., "Training with growing sets: A simple alternative to curriculum learning and self paced learning,” 2018. [Online]. Available: https: //openreview.net/forum?id=SJ1fQYlCZ

${ }^{4}$ Menegola, A., et al., "RECOD Titans at ISIC Challenge 2017," arXiv, abs/1703.04819, 2017.
} 